\title{
MacV: VCSELs for miniature atomic clocks
}

\author{
D. Zaouris, M. Knapp, M. Haji*, P. Gill \\ Time and Frequency Metrology \\ National Physical Laboratory \\ Teddington, UK \\ *mohsin.haji@npl.co.uk
}

\author{
I. Eddie, A. McKee \\ CST Global Ltd \\ Glasgow, UK
}

\author{
S. Shutts, P. M. Smowton \\ School of Physics and Astronomy \\ Cardiff University \\ Cardiff, UK
}
W. Meredith, D. Powell
Compound Semiconductor Centre Cardiff, UK

\begin{abstract}
Compact coherent population trapping (CPT) based clocks require single-mode, low-power consumption, high-speed and polarization-stable laser sources. We report our progress in developing customized vertical cavity surface emitting laser (VCSEL) diodes designed for operating on the D1 transition of cesium, and specifically tailored for CPT-based atomic clocks. The VCSELs provide high power $(>1 \mathrm{~mW})$, narrow linewidths $(<100$ MHz), mode-hop free tunability over $8 \mathrm{~nm}$, and are shown to be polarization stable over a wide range of operating bias conditions (average polarization suppression $>15 \mathrm{~dB}$ ). Preliminary spectroscopy has been observed.
\end{abstract}

Keywords-single mode VCSELs; polarization stable lasers; miniature atomic clocks; coherent population trapping

\section{INTRODUCTION}

Coherent population trapping (CPT) atomic clocks have been well-researched over the past two decades [1-2]. Compact CPT based clocks often employ vertical cavity surface emitting laser (VCSEL) diodes due to their low power, narrow linewidth, and exceptional reliability and lifetime properties [35]. Due to the relatively niche market opportunity for VCSEL manufacturers, VCSELs operating at the D1 transitions of cesium or rubidium are scarcely available commercially. Those which are available often have broad linewidths and operate at a temperature range that is incompatible with the high operating temperature within a physics package $\left(>70^{\circ} \mathrm{C}\right)$. As a result, we have developed a new capability in VCSELs tailored for CPT based atomic clocks, using customized epitaxy grown to accommodate $894.5 \mathrm{~nm}$ lasing at high temperatures, with chip designs enabling narrow-linewidth, single-mode, stable polarization and high-speed modulation. Some early results are presented here.

\section{VCSEL PRODUCTION AND TESTING}

Customized 4" GaAs/AlGaAs epitaxial wafers were grown, targeting a room temperature photoluminescence peak wavelength of $882 \mathrm{~nm}$ and a room temperature etalon specification at $892 \mathrm{~nm}$. VCSELs were fabricated on these wafers with unique designs enabling high-speed operation, single-mode, narrow-linewidth lasing with stable polarization. The VCSELs were pre-screened for their light-current-voltage

We acknowledge Innovate UK financial support
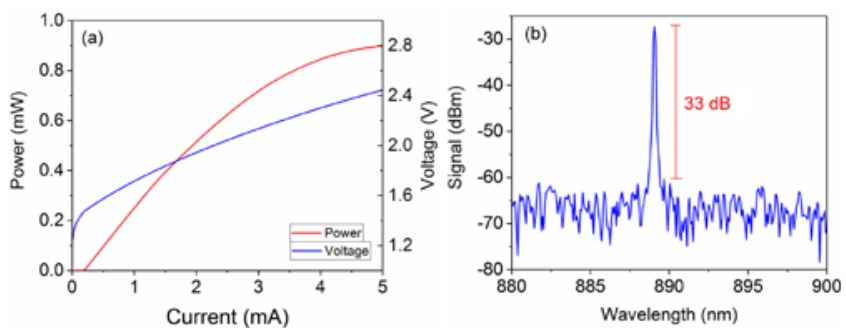

Fig. 1. (a) LIV characteristics of the VCSELs, and (b) example of a single mode spectrum indicating the side mode suppression of $33 \mathrm{~dB}$.

(LIV) and spectral characteristics prior to being packaged in a TO-46 can. The package also contains an integrated thermistor and Peltier thermo-electric control (TEC). Once packaged, the diodes were placed in a temperature controlled stage and characterized for spectral tuning, linewidth, optical intensity, polarization stability, $3 \mathrm{~dB}$ bandwidth, and linear spectroscopy through cesium atoms.

\section{RESULTS}

A low-noise current driver ( $<40 \mathrm{nA}$ RMS at $2 \mathrm{~mA}$ ) was used to characterize the VCSELs. The current-wavelength tuning coefficient was $0.88 \mathrm{~nm} / \mathrm{mA}$ and the temperature-wavelength tuning coefficient was $0.07 \mathrm{~nm} / \mathrm{K}$. The optical intensity at 70 ${ }^{\circ} \mathrm{C}$ reached a maximum of $0.9 \mathrm{~mW}$ at $5 \mathrm{~mA}$, at which point the laser intensity began to roll over (fig. 1a). An optical spectrum is shown in fig. 1b, indicating a side-mode suppression $>30 \mathrm{~dB}$.

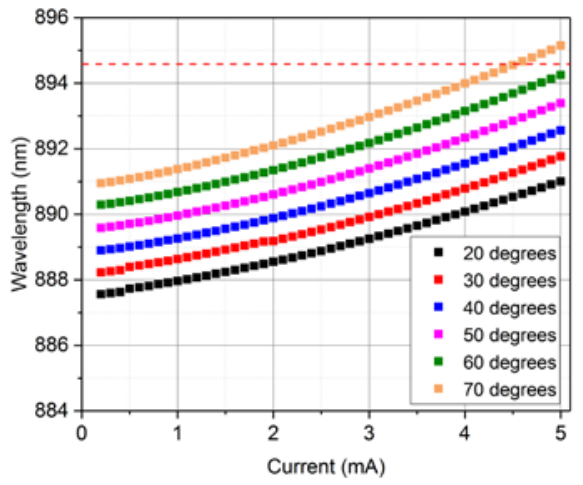

Fig. 2. Emission wavelength versus current at different temperatures. 
Single mode $894.5 \mathrm{~nm}$ lasing at $70^{\circ} \mathrm{C}$ was achieved using $\sim 4.5$ $\mathrm{mA}$ drive current (fig. 2). The VCSELs linewidth was $\sim 80$ $\mathrm{MHz}$, measured using the delay-line self-heterodyne technique. Devices with linewidths $<50 \mathrm{MHz}$ have also been measured.

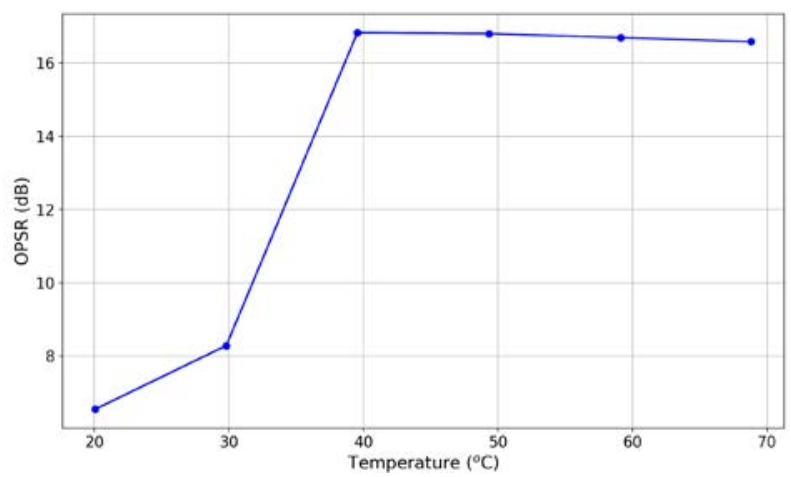

Fig. 3. OPSR versus temperature. Each point indicates the average OPSR over all applied drive currents $(0-5 \mathrm{~mA})$.

To determine the polarization stability of the VCSELs, a polarizing beam splitter was used to split the optical output of the lasers by its polarization state (TE and TM), each recorded by two photodetectors as a function of applied current and varying operating temperatures [6]. The dependence of the orthogonal polarization extinction ratio (OPSR) on the temperature is shown in Fig. 3, which indicates the average OPSR over all drive current (0-5 mA). An OPSR $>15 \mathrm{~dB}$ was achieved at temperatures in the operating range of $40^{\circ} \mathrm{C}$ to 70 ${ }^{\circ} \mathrm{C}$ (measurements were taken between $20^{\circ} \mathrm{C}$ and $70{ }^{\circ} \mathrm{C}$ in 10
${ }^{\circ} \mathrm{C}$ steps), indicating strong polarization stability over the measured range.

\section{FUTURE WORK}

Further iterations of manufacturing are in process, with improved epitaxial designs to promote fewer losses in the gain region as well as refinements to the etalon. VCSEL chip designs have been modified to improve the single-mode yield as well as improve the linewidth. Further tests are under way to characterise these VCSELs in a CPT atomic clock test bed.

\section{REFERENCES}

[1] S. Knappe, V. Shah, P.D.D. Schwindt, L. Hollberg, and J. Kitching, "A microfabricated atomic clock,” Appl. Phys. Lett., 2004, 85, pp. 14601462

[2] J. Kitching, “Chip-scale atomic devices,” Appl. Phys. Rev., 2018, 5, pp. 1-38.

[3] F. Gruet, A. Al-Samaneh, E. Kroemer, L. Bimboes, D. Miletic, C. Affolderbach, D. Wahl, R. Boudot, G. Mileti, R. Michalzik, "Metrological characterisation of custom designed $894.6 \mathrm{~nm}$ VCSELs for miniature atomic clocks," Optics Express, 2013, 21, pp. 5781-5792.

[4] E. Kroemer, J. Rutkowski, V. Maurice, R. Vicarini, M. Abdel Hafiz, C. Gorecki, R. Boudot, "Characterisation of commercially available vertical cavity surface-emitting lasers tuned on the Cs D1 line at $894.6 \mathrm{~nm}$ for miniature atomic clocks,” Applied Optics, 2016, 55, pp. 8839-8847.

[5] K.J. Ebeling, R. Michalzik, and H. Moench, "Vertical-cavity surfaceemitting laser technology applications with focus on sensors and threedimensional imaging,” Jpn. J. Appl. Phys., 2018, 57, pp. 1-11.

[6] J.M. Ostermann and R. Michalzik, "Polarisation control of VCSELs" in Springer Series in Optical Sciences, vol. 166, Springer, Berlin, Heidelberg, 2013, pp. 147-179. 